\title{
QuantEYE, the quantum optics instrument for OWL
}

\author{
C. Barbieri ${ }^{1}$, V. Da Deppo ${ }^{2}$, M. D'Onofrio ${ }^{1}$, D. Dravins ${ }^{3}$, \\ S. Fornasier ${ }^{1}$, R.A.E. Fosbury ${ }^{4}$, G. Naletto ${ }^{5}$, R. Nilsson ${ }^{3}$, \\ T. Occhipinti ${ }^{5}$, F. Tamburini ${ }^{1}$, H. Uthas ${ }^{3}$ and L. Zampieri ${ }^{6}$ \\ ${ }^{1}$ Department of Astronomy, University of Padova, Vicolo dell'Osservatorio, 2, I-35122 Padova, \\ Italy, email: cesare.barbieri@unipd.it \\ ${ }^{2}$ CNR-INFM LUXOR, c/o Department of Information Engineering, Padova, Italy \\ ${ }^{3}$ Lund Observatory, Lund, Sweden \\ ${ }^{4}$ ESA/ST-ECF, Garching bei München, Germany \\ ${ }^{5}$ Department of Information Engineering, University of Padova, Padova, Italy \\ ${ }^{6}$ INAF Astronomical Observatory of Padova, Italy
}

\begin{abstract}
A brief description of the QuantEYE instrument proposed as a focal plane instrument for OWL is given. This instrument is dedicated to the very high speed observation of many active phenomena with a photon counting capability of up to $1 \mathrm{GHz}$. The system samples the beam in $10 \times 10$ subpupils, each focused on a fast photon counting detector.
\end{abstract}

Keywords. instrumentation: photometers - methods: statistical, telescopes.

\section{Introduction}

QuantEYE is the conceptual study for a Quantum Optics instrument, developed in the frame of studies for the focal plane instrumentation of the ESO OWL (OverWhelmingly Large) telescope. This instrument could be applied to any Extremely Large Telescope of the future to open a new window for the study of the Universe. Its scientific motivations are detailed in the work by Dravins et al. (2006); in the following a brief description of its optics characteristics and of the detector system is given.

QuantEYE is meant to measure the photon second- and higher-order correlation functions providing the highest time resolution (1ns or better) of all astronomical instruments (quantum effects are fully developed over the inverse optical bandwidth: $0.1 \mathrm{~nm}$, namely $10^{11} \mathrm{~Hz}$, corresponding to $10 \mathrm{ps}$ ). Furthermore, it will permit to carry out very high timeresolution studies beyond those feasible with 8-10 m class telescopes.

\section{Instrument characteristics}

The QuantEYE instrument consists of an inverse Cassegrain telescope which collimates the light beam after the OWL focus (see Fig. 1). At this position of the radiation beam, which has an annular shape of about $100 \mathrm{~mm}$ maximum radius, the possibility of inserting filters and/or polarizers is foreseen. The filtered beam is then collected by a $10 \times 10$ lenslet array sampling the instrument pupil. Each lenslet is a compound system of two doublets having a square section of $1 \mathrm{~cm}$ side, adopting only spherical surfaces for ease of fabrication and with an $\mathrm{f} / 1$ aperture. At the focus of each lens an optical fiber leads the photons to Single Photon Avalanche Detectors (SPADs). A second moving detector head, which can span the whole 3 arcmin scientific field of view of OWL, has also been foreseen to simultaneously observe a comparison star. 


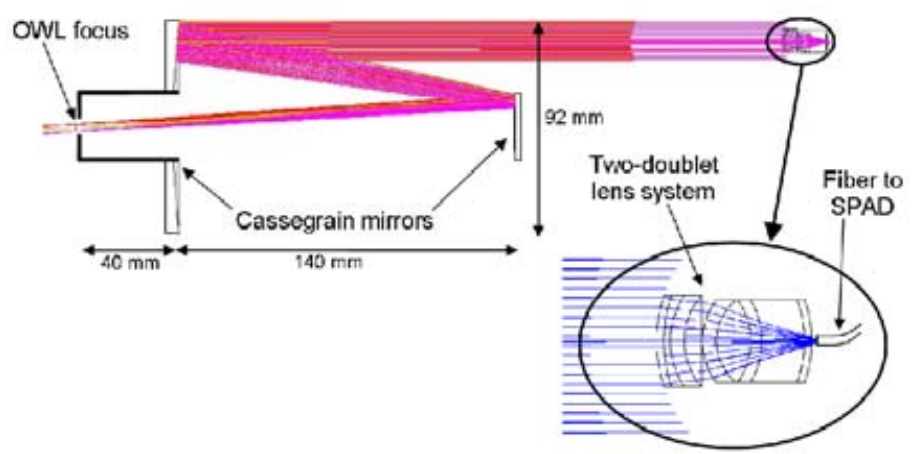

(a)

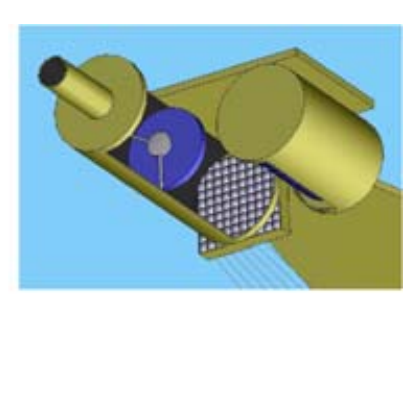

(b)

Figure 1. Sketch of the optical concept for QuantEYE. (a) The collimator-lenslet system magnifies $1 / 60$ times (collimator focal length $=600 \mathrm{~mm}$, lens focal length $=10 \mathrm{~mm}$ ), giving a nominal spot size of $50 \mu \mathrm{m}$ for a 1-arcsec source. For sake of simplicity, only one lenslet of the whole $10 \times 10$ array is shown. (b) A cut 3D view of the system (excluding the detectors) is shown.

The instrument working spectral range is 400-900nm. In this spectral region SPADs exist with a quantum efficiency better than $40 \%$, with very good timing accuracy (better than $100 \mathrm{ps}$ ) and with very low dark count rate (of the order of $100 \mathrm{c} / \mathrm{s}$ ): putting together the 100 SPADs, a photon counting rate up to $1 \mathrm{GHz}$ can be obtained. The arrival time of each photon is acquired and stored, and then given as input to an asynchronous post processing which guarantees data integrity for the following scientific investigation. Also an on-line correlator is included, to allow real time control of some particular SPADs. Single Photon Detectors coupled to a precise time reference given by a Hydrogen Maser clock allow continuous photon counting with a time tagging precision better than 100ps for several hours.

The choice of this optical design is mainly due to the present day technological limitation as SPAD arrays (of contiguous pixels) do not exist. With the proposed solution, this limitation is overcome, greatly limiting the dead time problem, and so providing a fixed-area very high speed photometer with a tremendous dynamic range, from $5^{\text {th }}$ to the $30^{\text {th }}$ magnitude. In addition, it is likely that in the next few years the development of new detectors and new optical clocks can push the time resolution into the picosecond region for several hours of continuous operation. Quantum computing and quantum algorithms might help in the analysis of the huge, multidimensional data base generated by QuantEYE.

\section{Conclusions}

The QuantEYE instrument proposed as a focal plane instrument of OWL has been briefly described. This instrument is dedicated to the very high speed observation of many active phenomena with a photon counting capability up to $1 \mathrm{GHz}$. The optical design envisaged for this instrument allows to overcome the present technological limitation of the detector speed, but in the near future with the possibility of using SPAD arrays it is reasonable to think to simpler optical designs.

\section{Reference}

Dravins, D., Barbieri, C., Fosbury, R.A.E., Naletto, G., Nilsson, R., Occhipinti, T., Tamburini, F., Uthas, H., Zampieri, L. 2006, this conference. 